\section{Pulsed Radiofrequency Ablation for Anterior Cutaneous Nerve Entrapment Syndrome: A Case Report \\ Susheel Govindan, $\mathrm{MD}^{1}$, Christopher Lam, MD'1, Usman Latif, MD, MBA ${ }^{1}$ \\ 'The University of Kansas Health System, Depart- ment of Anesthesiology - Kansas City, Kansas}

\section{ABSTRACT}

Anterior Cutaneous Nerve Entrapment Syndrome (ACNES) is a cause of chronic abdominal pain in which nerves become pathologically entrapped and irritated, leading to pain. The prevalence of the syndrome is 15 to 30 percent, and many treatment approaches begin with pharmacologic therapy. If pharmacologic therapy fails, trigger point injections, nerve blocks, and neurectomy are subsequent therapeutic options. We present the case of a 50-year-old female with chronic abdominal pain and steroid allergy who presented to the pain clinic after failed mainstay therapy including gabapentin, amitriptyline, nortriptyline, duloxetine, NSAID, and acetaminophen. She was diagnosed with Anterior Cutaneous Nerve Entrapment Syndrome (ACNES). A primary intervention of Bilateral Transversus Abdominis Plane (TAP) Block with local anesthetic provided $80 \%$ pain relief. Following successful primary intervention, a secondary intervention of Pulsed Radiofrequency Ablation (PRF) provided pain relief for 2 months.

\section{Glossary of Terms:}

ACNES - Anterior Cutaneous Nerve Entrapment Syndrome TAP - Transversus Abdominis Plane

PRF - Pulsed Radiofrequency Ablation

RFA - Radiofrequency Ablation

\section{Keywords: Nerve Entrapment, Abdominal Pain.}

\section{Introduction}

Abdominal pain and associated gastrointestinal diseases utilize a significant portion of healthcare resources in the United States. It is estimated that abdominal pain accounts for 5 to $10 \%$ of all chief complaints in the emergency department, and that healthcare expenditures nationwide can exceed $\$ 10$ billion annually., ${ }^{1,2}$ Therefore, adequately diagnosing and treating abdominal pain can have a dramatic impact in overall public health. Many etiologies behind abdominal pain exist, including hepatobiliary, cardiac, gastric, pancreatic, colonic, and genitourinary pathologies. Moreover, characterization of the pain itself can provide insight into causation. For example, visceral nerve fibers that innervate organs can cause poorly-localized, achy pain. In contrast, somatic fibers that innervate the peritoneum may cause sharp, well-localized pain. ${ }^{1}$

Another factor important to the appropriate treatment of abdominal pain is the chronicity of the problem. The approach to chronic undiagnosed abdominal pain starts with an appropriate history and physical exam. Further clues to diagnosis can be found in location of pain, particularly the difference in etiologies behind localized and diffuse abdominal pain. Diffuse abdominal pain can be pathologically derived from disorders such as centrally mediated abdominal pain syndrome (CAPS), endometriosis, narcotic bowel syndrome, Ehlers Danlos Syndrome, mast cell activation syndrome, and chronic mesenteric ischemia. On the other hand, localized pain can be further differentiated using the Carnett's Sign. Localized abdominal pain with a negative Carnett's Sign can be indicative of functional dyspepsia or irritable bowel syndrome, while a positive Carnett's Sign can point to chronic abdominal wall pain. ${ }^{3}$

Chronic abdominal wall pain, also known as Anterior Cutaneous Nerve Entrapment Syndrome (ACNES), is a cause of abdominal pain in which thoracic intercostal nerves are trapped and irritated by abdominal pressure or postoperative scar tissue formation. Anatomically, as thoracic intercostal nerves exit the spinal neuroforamina, subcostal nerves can become trapped between the internal oblique and transversus abdominis, while lateral cutaneous nerves can become trapped between subcutaneous tissue and the external oblique. ${ }^{4}$ The prevalence of the syndrome ranges between 15 and 30 percent, with the most common age group affected being those between 30 and 50 years of age. ${ }^{5}$ It is the underlying diagnosis in $2 \%$ of patients presenting to the emergency department with abdominal pain, and $10 \%$ of chronic abdominal pain cases in the outpatient setting. ${ }^{6}$ Patients with ACNES traditionally present with well-localized pain, with an increase in tenderness to palpation during muscle tensing. Right upper quadrant pain is the presentation in $40 \%$ of patients. ${ }^{6}$ Furthermore, patients often present with a positive Carnett's sign. To test this sign, a patient lies supine while the provider places a finger on the painful site. The patient raises both legs off of the table, or solely their head off of the table, contracting abdominal muscles. This maneuver can worsen ACNES symptoms, leading to a positive Carnett's sign. ${ }^{7}$ Along with a positive Carnett's sign, the diagnostic criteria for ACNES include well-localized abdominal pain and a response to trigger point injection of local anesthetic. Recent retrospective studies have shown 
that pseudovisceral symptoms, including a change in defecation habits, appetite suppression, nausea, and bloating can be associated with ACNES. ${ }^{8}$ Current treatment options include pharmacologic therapy, trigger point injections, ultrasound-guided nerve blocks, chemical neurolysis, and surgical neurectomy. ${ }^{5}$

We present a case of a 50-year-old female with chronic abdominal pain who was diagnosed with ACNES. After failed mainstay pharmacologic therapy, and with consideration to the patient's allergy to corticosteroids, a bilateral Transversus Abdominis Plane (TAP) Block followed by Pulsed Radiofrequency Ablation (PRF) were performed. The patient had 2 months of pain relief following this intervention. The manuscript adheres to the applicable EQUATOR guideline. HIPAA authorization was obtained from the patient to publish this case report.

\section{Case Report}

A 50-year-old female with a past medical history of deep vein thrombosis, pulmonary embolism, hypertension, hypothyroidism, gastroesophageal reflux disease, and depression presented to the pain clinic in 2019 with a chief complaint of chronic abdominal pain, recurrent since 2003. Her only surgery was a cholecystectomy in 1996. She had a syncopal allergy to steroids, further complicating her clinical course due to intolerance of steroid-based therapies. She presented with right upper quadrant abdominal pain, worse with eating, that lasted five to six hours with each episode. Along with her symptoms, she also noted weight loss and intermittent vomiting since the pain started. Her history was significant for multiple visits to gastrointestinal specialists since 2003, resulting in upper and lower endoscopies, ERCP, and a gastric emptying study. She underwent laparoscopy for lysis of adhesions in 2003 and 2011, which provided minimal relief. All CT scans and endoscopies were negative for pathology. One gastrointestinal specialist noted right rib tenderness on physical exam, and leading differentials included musculoskeletal pain, mesenteric ischemia, functional bowel disorder, and radiculopathy. Barium esophagram, endoscopic ultrasound, and mesenteric dopplers came back negative. A lower esophageal injection of botox for possible achalasia provided no relief. Moreover, laboratory workup, including enzymatic analysis during an episode of pain, were negative. The patient tried a variety of pharmacologic therapies such as acetaminophen, non-steroidal anti-inflammatory drugs, gabapentin, amitriptyline, nortriptyline, and duloxetine. The only medication that provided relief to the patient was cyclobenzaprine, and the relief was minimal. Exercise and physical therapy also proved to be ineffective.

Physical exam at the time of presentation to the chronic pain clinic showed epigastric and right upper quadrant tenderness, guarding, and a positive Carnett's sign. Due to the minimal effect of pharmacologic therapy alone, and with her pain approaching midline, the team opted for a primary intervention with a bilateral TAP Block using a l:1 solution of $2 \%$ lidocaine and $0.5 \%$ bupivacaine. Using ultrasound guidance in an anterolateral plane, a 22-guage, 3.5 inch spinal needle was advanced to the border between the internal oblique and transversus abdominis muscle. After negative aspiration, the solution was injected. The patient had $80 \%$ pain relief for thirty hours following the procedure.

After a successful primary intervention for short-term pain relief, the patient opted to pursue an additional therapy with the goal of long-term pain relief. After discussion with the patient, the team performed a secondary intervention with PRF, using $10 \mathrm{~mL}$ of $0.5 \%$ bupivacaine and $2 \mathrm{~mL}$ of $1 \%$ lidocaine prior to ablation. Using ultrasound, 2 needles were inserted at different locations in the fascial plane, between the internal oblique and transversus abdominis (Figure 1). Following injection of bupivacaine, PRF was performed using an Interventional Spine RF Generator System, $1 \mathrm{MHz}$, at $42{ }^{\circ} \mathrm{C}$ for 120 seconds. A 100 MM curved, 10 MM, 18 gauge radiofrequency cannula was used to perform the ablation. The patient had pain relief for the following two months after the procedure.

\section{Discussion}

ACNES has both non-invasive and invasive treatment modalities. Although previous literature has indicated up to $42 \%$ of patients having complete pain remission after injection of local anesthetic into the abdominal wall alone, patients with persistent pain are forced to search for alternative methods of pain relief., ${ }^{910}$ The addition of adjuvants to local anesthetic has been attempted for longer duration of pain relief. One such adjuvant, dexamethasone, has been shown to prolong the effect of long-acting anesthetics up to 1,306 minutes. ${ }^{11,12}$ If pain continues after local anesthetic and adjuvant injection, neurectomy use has been shown to provide pain relief in up to $90 \%$ of patients. ${ }^{13}$ However, the lasting nerve damage associated with neurectomy has led to the search for less destructive methods of pain management. One such method with recent utilization is PRF for ACNES.

PRF uses high-frequency intermittent current with tissue temperatures below $42^{\circ} \mathrm{C}$ to stun nerves and prevent long-term neuronal damage. ${ }^{14,15}$ In terms of efficacy, a clinical 
trial recently showed PRF to be a minimally invasive treatment alternative to neurectomy in patients with ACNES. ${ }^{16}$ In the case presented, the patient had a notable allergy to corticosteroids, resulting in the search alternative treatment options. Before attempting PRF for long-lasting pain relief, a treatment of shorter duration was used to determine the location of nerve irritation and efficacy of nerve disruption. After the patient received substantial short-term pain relief following TAP Block, a long-acting therapy was initiated. The alternative option of neurolysis was considered for intervention, but the concern of post-procedure neuritis and Deafferentation Pain Syndrome supported the choice of PRF. Moreover, the patient's allergy to steroids would have made the prophylaxis and treatment of neurolysis-associated complications difficult to address.

In regards to hydrodissection prior to $\mathrm{PRF}$, it is unlikely that the local anesthetic provided prolonged pain relief. Previous studies have even shown that hydrodissection with saline or local anesthetic is safe and effective in reducing surrounding tissue damage when combined with RFA. ${ }^{17}$ Additional studies have implicated that pre-ablation local anesthetic injection may improve pain scores in the early post-operative period, justifying clinical usage. ${ }^{18}$ Therefore, attributing this episode of sustained pain relief solely to pre-RFA local anesthetic is not supported by clinical evidence. Unfortunately, the patient's pain returned following two months of relief. Currently, a more permanent treatment approach is being explored, with the possibility of future peripheral nerve stimulator implantation.

\section{Appendix}

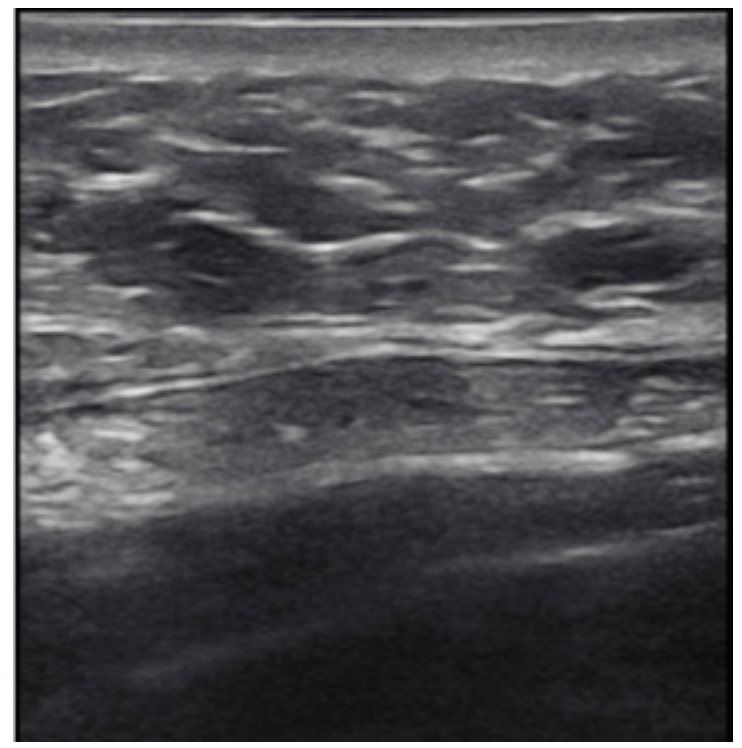

Figure 1: Ultrasound capturing PRF between the internal oblique and transversus abdominis.

\section{Corresponding author:}

Susheel Govindan MD

Email: Sgovindan2@kumc.edu

\section{References}

${ }^{1}$ Natesan S, Lee J, Volkamer H, Thoureen T. EvidenceBased Medicine Approach to Abdominal Pain. Emerg Med Clin North Am. 2016;34(2):165-190. PMID: 27133239

${ }^{2}$ Peery AF, Crockett SD, Murphy CC, et al. Burden and Cost of Gastrointestinal, Liver, and Pancreatic Diseases in the United States: Update 2018. Gastroenterology. 2019;156(1):254-272.e211. PMID: 30315778

${ }^{3}$ Pichetshote N, Pimentel M. An Approach to the Patient With Chronic Undiagnosed Abdominal Pain. Am J Gastroenterol. 2019;114(5):726-732. PMID: 30694864

${ }^{4}$ Birthi P, Calhoun D, Grider JS. Pulsed radiofrequency for chronic abdominal pain. Pain Physician. 2013;16(4):E443-445. PMID: 23877469

${ }^{5}$ Chrona E, Kostopanagiotou G, Damigos D, Batistaki C. Anterior cutaneous nerve entrapment syndrome: management challenges. J Pain Res. 2017;10:145-156. PMID: 28144159

${ }^{6}$ Kamboj AK, Hoversten P, Oxentenko AS. Chronic Abdominal Wall Pain: A Common Yet Overlooked Etiology ofChronic Abdominal Pain. Mayo Clin Proc. 2019;94(1):139144. PMID: 30611441

7 Takada T, Ikusaka M, Ohira Y, Noda K, Tsukamoto T. Diagnostic usefulness of Carnett's test in psychogenic abdominal pain. Intern Med. 2011;50(3):213-217. PMID: 21297322

${ }^{8}$ Mol FMU, Maatman RC, De Joode L, Van Eerten P, Scheltinga MR, Roumen R. Characteristics of 1116 Consecutive Patients Diagnosed With Anterior Cutaneous Nerve Entrapment Syndrome (ACNES). Ann Surg. 2019. PMID: 30817351

${ }^{9}$ Watari T, Tokuda Y. Anterior cutaneous nerve entrapment syndrome. BMJ Case Rep. 2019;12(12). PMID: 31796444

${ }^{10}$ Mol FMU, Maatman RC, De Joode LEGH, Van Eerten P, Scheltinga MR, Roumen R. Characteristics of 1116 Consecutive Patients Diagnosed With Anterior Cutaneous Nerve Entrapment Syndrome (ACNES). Ann Surg. 2019. PMID: 30817351

${ }^{11}$ Choi S, Rodseth R, McCartney CJ. Effects of dexamethasone as a local anaesthetic adjuvant for brachial plexus block: a systematic review and meta-analysis of randomized trials. Br J Anaesth. 2014;112(3):427-439. PMID: 24413428

${ }^{12}$ Krishna Prasad GV, Khanna S, Jaishree SV. Review of adjuvants to local anesthetics in peripheral nerve blocks: 
Current and future trends. Saudi J Anaesth. 2020;14(1):77-

84. PMID: 31998024

${ }^{13}$ van Assen T, Boelens OB, van Eerten PV, Scheltinga MR, Roumen RM. Surgical options after a failed neurectomy in anterior cutaneous nerve entrapment syndrome. World J Surg. 2014;38(12):3105-3111. PMID: 25189442

${ }^{14}$ Ahadian FM. Pulsed radiofrequency neurotomy: advances in pain medicine. Curr Pain Headache Rep. 2004;8(1):34-40. PMID: 14731381

${ }^{15}$ Sluijter M. The Effects of Pulsed Radiofrequency Fields Applied to the Dorsal Ganglia - a Preliminary Report. Pain Clinician. 1998;11:109-117.

${ }^{16}$ Maatman RC, van Kuijk SMJ, Steegers MAH, et al. A Randomized Controlled Trial to Evaluate the Effect of Pulsed Radiofrequency as a Treatment for Anterior Cutaneous Nerve Entrapment Syndrome in Comparison to Anterior Neurectomy. Pain Practice. 2019;19(7):751-761. PMID: 31188514

${ }^{17}$ Cui D, Ding M, Tang X, et al. Efficacy and safety of a combination of hydrodissection and radiofrequency ablation therapy for benign thyroid nodules larger than $2 \mathrm{~cm}$ : A retrospective study. J Cancer Res Ther. 2019;15(2):386-393. PMID: 30964116

${ }^{18}$ Arıcı T, Kurçaloğlu M, Köken İ, Uzuner B, Eyigör C, Uyar M. The effects of preadministration of local anesthetic on pulsed radiofrequency. Turk J Med Sci. 2018;48(3):462468. PMID: 29914237 